\title{
Effectiveness and Social Validity of FBAs for Youth At-Risk or With High Incidence Disabilities: A Meta-Analysis
}

\author{
John W. Maag ${ }^{1}$ \\ ${ }^{1}$ Department of Special Education and Communication Disorders, University of Nebraska-Lincoln, USA \\ Correspondence: John W. Maag, Department of Special Education and Communication Disorders, 202 Barkley \\ Memorial Center, University of Nebraska-Lincoln, Lincoln, NE 68583-0732, USA. Tel: 402-472-5477. E-mail: \\ jmaag1@unl.edu
}

\author{
Received: January 24, 2019 Accepted: February 16, 2019 Online Published: February 28, 2019 \\ doi:10.5539/jedp.v9n1p41 URL: http://doi.org/10.5539/jedp.v9n1p41
}

\begin{abstract}
This meta-analysis examined the effectiveness and social validity of 44 functional behavioral assessment (FBA) studies using single case research designs (SCRDs) conducted with youth displaying challenging behaviors or had high incidence disabilities. Three effect sizes were calculated: standard mean difference (SMD), Tau- $U$, and improvement rate difference (IRD). Fisher's conservative dual criterion (CDC), which is a statistical aid to visual analysis, was also applied. Social validity was assessed by using indicators described by Kazdin (2010). Effect sizes were in ranges indicating moderate to large effects. Approximately $71 \%$ of $\mathrm{AB}$ contrasts reflected $\mathrm{CDC}$ systematic change. However, only $44 \%$ of studies assessed social validity. There were no significant differences in effectiveness of interventions whether or not a functional analysis was conducted nor whether the controlling function was escape or attention. Results are discussed in terms of FBA implementation issues related to social validity and the necessity for conducting a functional analysis for these youth.
\end{abstract}

Keywords: disabilities, FBAs, meta-analysis, social validity, youth at-risk

\section{Introduction}

Applied Behavior Analysis (ABA) is a performance-based self-evaluative method for changing behavior, and whose dimensions were described by Baer, Wolf, and Risley (1968) approximately half a century ago in the first issue of the Journal of Applied Behavior Analysis. There have been many important developments in the ABA literature since then, but the issue of social validity and the development of functional behavioral assessment (FBA) arguably have been the most instrumental (Maag, 2014). Ten years after Baer and his colleagues wrote their seminal article, Wolf' (1978) wrote another influential article on social validity which addresses whether a relevant audience (e.g., educators, mental health providers) find interventions in real-life settings to be acceptable in terms of their goals, methods, outcomes, and ease of implementation. Around that time, Carr (1977) described how self-injurious behaviors resulted from either positive reinforcement or negative reinforcement. Based on his hypotheses, Iwata, Dorsey, Slifer, Bauman and Richman (1982) conducted what many consider the first study on FBA.

FBA refers to a series of heuristic approaches for determining the purpose (i.e., source of environmental reinforcement) youths' challenging behaviors serve. An important byproduct of an FBA is the development of a behavior intervention plan (BIP) that addresses the identified function(s). It is believed that the most effective interventions implemented in school and clinical settings are those based on the purpose maladaptive behaviors serve (Ervin et al., 2001). There have literally been hundreds of studies conducted on various aspects of FBA methodologies across different participant characteristics, and approximately 17 systematic reviews have been conducted on various procedures and populations - eight of which used meta-analytic approaches to calculate effect sizes (Bruni et al., 2017; Common, Lane, Pustejovsky, Johnson, \& Johl, 2017; Delfs \& Campbell, 2010; Gage, Lewis, \& Stichter, 2012; Goh \& Bambara, 2012; Losinski, Maag, Katsiyannis, \& Ennis, 2014; McKenna, Flower, Kim, Ciullo, \& Haring, 2015; Miller \& Lee, 2013). Some of the variables addressed in these reviews included, but were not limited to, single case synthesis, effect size approaches, different populations, quality of studies, and positive supports in schools and clinics.

One area that has received little attention in the synthesis of FBA literature is evaluating the social validity of interventions based on this approach (Broussard \& Northrup, 1997; Clarke et al., 1995; McKenna, Flower, \& 
Adamson, 2016; Umbreit, 1995). A commonly held belief by practitioners is that FBAs are complicated, multifaceted, and time-consuming. Fuchs, Fuchs, and Bahr (1990) stated that approaches which involve intense effort, extended time periods, or expertise not normally available may not be embraced by many educators in general education settings. There is some evidence that general education teachers can be taught how to conduct an FBA (e.g., Maag \& Larson, 2004; Moore et al., 2002; Packenham, Shute, \& Reid, 2004), but whether they use this methodology independently, consistently, and with fidelity has not been directly examined.

Although there have been many systematic reviews over various aspects and participants of FBA, many focus on preschool children or those children with moderate to severe intellectual or developmental disabilities or with autism spectrum disorders. In addition, many reviews included studies conducted in clinic, residential, psychiatric, or home settings but only a few focused on students in K-12 grades that are either at-risk or have high incidence disabilities or display challenging behaviors in classroom settings. One such review was undertaken by Common et al. (2017). However, their review only focused on 18 studies between 2007 and 2015 that used the function matrix and function-based intervention model developed by Umbreit, Ferro, Liaupsin, \& Lane (2007).

The purpose of the current meta-analysis was to expand on Common et al. (2017) by reviewing the corpus of FBA studies beginning in 1982 when, arguably, the first FBA study was conducted by Iwata and colleagues. A second purpose was to evaluate the extent to which studies addressed social validity in terms of assessing the acceptability of interventions based on a FBA by the interventionist implementing them and the methods for collecting this information.

\section{Method}

\subsection{Study Identification and Selection}

A systematic search of the FBA literature with youth at-risk or with high incidence disabilities was conducted using Academic Search Premier (EBSCO) search source with PsychINFO, ERIC, and MEDLINE as the selected data bases. An archival search was conducted from the references of studies included in previous reviews. Studies had to be in English and published in peer-reviewed journals between January 1, 1982 and January 1, 2017. Only studies in which an FBA resulted in a function-based intervention were included (i.e., studies that only manipulated variables during a functional analysis but did not result in an intervention were excluded). Search terms included all possible combinations and derivations of the following: functional assessment, FBA, functional analysis, high incidence disabilities, students at risk, learning disabilities, emotional behavioral disorder, attention deficit hyperactivity disorder, oppositional defiant disorder, conduct disorder, speech/language impairment, other health impaired.

Initial screening consisted of reading titles and abstracts of 423 obtained studies by the researcher and graduate assistant to determine if they should be read in their entirety. Of these studies, 411 were read to determine if they met the following inclusion criteria: (a) the study involved youth who either had high incidence disabilities, at risk that displayed challenging behaviors, or had either attention deficit hyperactivity disorder, conduct disorder, or oppositional defiant disorder $(n=99)$, (b) the sample included youth from grades k-12 $(n=93)$, (c) studies that were conducted in school classroom settings $(n=83)$, (d) studies developed and assessed an intervention based on the FBA conducted $(n=53)$, (e) studies that targeted inappropriate behavior for the FBA $(n=44)$, and (e) studies employed a SCRD to analyze the FBA $(n=44)$. A total of 44 articles were included in the present review.

\subsection{Coding Procedures}

Articles retrieved from the search were coded along eight variables: (a) participant characteristics (age, grade, gender), (b) disability/diagnosis/at-risk, (c) setting (general education, special education), (d) design features (AB, reversal, multiple baseline, alternating treatments), (e) dependent variables, (f) type of FBA conducted based on the methods used, (g) obtained function and whether a functional analysis was conducted, and (h) intervention developed from the FBA. Studies were also coded for the presence or absence of four social validity indicators described by Kazdin (2010).

\subsection{Statistical Analysis}

Data extraction. Data were extracted from the graph(s) in each study using Enguage Digitizer (Mitchell, 2002) - an open source digitizing software package that converts graphic image files (e.g., .jpg, .bmp) into numerical data. Enguage is comparable to Biosoft's Ungraph 5.0 that was recommended in the manual developed by Nagler, Rindskopf, and Shadish (2008) for conducting SCRD meta-analysis.

Effect size calculations. Three types of effect sizes were calculated. Standard mean difference (SMD) was calculated because it is the SCRD analog of Cohen's (1988) $d$ and permits comparison to non-single-case methods. 
However, SMD is considered unreliable because of small number of observations and floor effects limiting variability which results in overestimates of the parametric treatment effects (Horner, Swaminathan, Sugai, \& Smolkowski, 2012; Scruggs \& Mastropieri, 2012). Therefore, a ceiling based on the $3^{\text {rd }}$ quartile was used to decrease overestimates (i.e., 3.40). Improvement rate difference (IRD) was computed because it provides an effect size similar to the risk difference used in medical treatment research which has a proven track record in hundreds of studies (Parker, Vannest, \& Brown, 2009). Finally Tau- $U$ values were computed because it controls for monotonic trend.

Additional analysis. Independent t-tests were computed to compare differences in effectiveness of study interventions that did and did not conduct a functional analysis to corroborate hypothesized functions. Independent t-tests were also computed to compare difference in effectiveness of study interventions between those developed based on escape versus attention functions. These t-tests were computed for all three effect size calculations.

Conservative dual criterion. The $\mathrm{CDC}$ lines were computed from $\mathrm{AB}$ contrasts (i.e., baseline and intervention adjacent phases) extracted from the graphs. Two lines were calculated: the mean line and the least squares regression line. Each line was dropped 0.25 standard deviations and then superimposed on the intervention data phase. Then the criteria developed by Fisher, Kelley, and Lomas (2003) were applied to determine whether changes in data were systematic or nonsystematic by examining the number of data points during the intervention phase that were below the 0.25 standard deviation least squares regression line. Intervention phases must consist of at least five data points in order to apply CDC lines.

\subsection{Social Validity}

Social validity was assessed for each study based on the four components described by Kazdin (2010). First, did researchers have a specifically stated goal related to social validity of the study? Second, was there a social comparison component? That is, were data collected on a peer(s) who did not display challenging behaviors on the dependent variables to determine if participants' intervention data were commensurate with the peers' level. Third, did the researchers include a social validity scale that assessed participating teachers' degree to which interventions were helpful, easy to implement, and outcomes were positive? Fourth, did researchers report on the level to which intervention addressed the specific goal?

\subsection{Publication Bias}

Publication bias, or the "file drawer" effect was addressed. This phenomenon refers to presence of potential bias existing because of a greater likelihood that published research shows positive findings (Rosenthal, 1979). In a meta-analysis of group design studies, the Meta-Win's Fail-Safe function (Rosenberg, Adams, \& Gurevitch, 2000) can be used to estimate the number of unpublished studies with null results sufficient to reduce observed effect sizes to a minimal level (i.e., $<.20$ ). However, there is no comparable formula in SCRD meta-analyses. Therefore, to reduce the likelihood of the "file drawer" effect, the number of cases with no effect were added to the group of study effect sizes to reduce the overall effect to insignificant or suspect levels $(d<.20$; $\operatorname{IRD}<37$; Tau $<.20)$.

\subsection{Inter-Rater Reliability}

Interrater reliability (IRR) data were conducted on 20 randomly selected articles out of the 44 included studies for a total of $45 \%$ of studies on the eight coded study characteristics. This percentage is congruent with other published SRCD systematic reviews (e.g., Maggin, Briesch, \& Chafouleas, 2013). Social validity IRR was conducted on all 44 studies to determine the presence or absence of the four indicators described previously. Interrater reliability was calculated both for study characteristics and social validity components by dividing the total number of agreements by the total number of agreements plus disagreements for each item and averaged for all items. The author and one doctoral-level graduate assistant coded the articles for all variables and IRR for study characteristics was $88 \%$ (range: $72 \%-100 \%$ ) and $85 \%$ (range $68 \%-100 \%$ ) for social validity.

\section{Results}

\subsection{Characteristics of Participants and Settings}

A total of 91 participants were included in the 44 studies contained in this analysis. Descriptions of participant age, gender, grade level (when stated) and disability/diagnosis/at-risk appear in Table 1. Participants' ages ranged from six years old (e.g., Grady \& Peck, 1997) to 15 years of age (Patterson, 2009) with a mean age of 8.86 years and a median age of 9 years. There were eight studies for a total of 14 participants (13 males, 1 female) that only reported grade level and not age, but together had a median of fourth grade. All studies reported gender with more males ( $n$ $=76)$ than females $(n=15)$ represented. The majority of participants in the studies were at-risk and displayed challenging behaviors but were not have a high incidence disability nor the psychiatric conditions described 
previously $(n=56)$ followed by participants identified as $\operatorname{EBD}(n=17)$, ADHD $(n=13)$, and LD $(n=5)$. Approximately $39 \%$ of the studies $(n=17)$ only had one participant.

The majority of studies were conducted in a general education classroom $(n=34)$. The next most common setting was identified as self-contained classroom $(n=8)$. There were two studies that identified the setting as "special education" (Bessett \& Wills, 2007; Clarke et al., 1995).

Table 1. Characteristics of Studies Reviewed

\begin{tabular}{|c|c|c|c|c|c|}
\hline Study & Participants & $\begin{array}{l}\text { Disability/ } \\
\text { diagnosis }\end{array}$ & Dependent variables & Obtained function & Intervention \\
\hline $\begin{array}{l}\text { 1. Bessett \& } \\
\text { Wills }(2007)\end{array}$ & 8-yr. old male & At-risk & $\begin{array}{l}\text { Inappropriate } \\
\text { verbalizations; } \\
\text { physical aggression }\end{array}$ & $\begin{array}{l}\text { Attention \& escape } \\
\text { (multiply } \\
\text { controlled) } \\
\text { (functional analysis) }\end{array}$ & $\begin{array}{l}\text { Replacement behavior (i.e., } \\
\text { raise hand for attention; ask for } \\
\text { a break for escape) }\end{array}$ \\
\hline
\end{tabular}

2. Broussard 4 boys; 2 7-yrs.

\& Northrup old \& 2 9-yrs. (1997)

2 ADHD, 2 verbalizations; out of Peer attention

Differential reinforcement of seat; playing with (functional analysis) other behavior (DRO) objects

3. Campbell 2 10-yr. old At-risk
\& Anderson
(2008)

Noncompliance,
disruption, negative Peer attention
verbal/physical
interaction, out of seat

Check-in/Check-out with contingent morning lunchroom seating choice
4. Carter \& Horner (2009) 3 5-to-7-yr. old boys At-risk At-risk Talk-outs; out of seat;
noncompliance;
aggression

Peer \& adult teac
attention; escape

First Step to Success; extinction; increased peer \& teacher attention for appropriate behaviors; modifying work \& preferred activities

\begin{tabular}{|c|c|c|c|c|c|}
\hline $\begin{array}{l}\text { 5. Cho \& } \\
\text { Blair }(2017)\end{array}$ & 13 -yr. old male & ADHD & $\begin{array}{l}\text { Leaving seat; saying } \\
\text { "no"; ignoring teacher } \\
\text { directions }\end{array}$ & $\begin{array}{l}\text { Teacher attention \& } \\
\text { escape (multiply } \\
\text { controlled) } \\
\text { (functional analysis) }\end{array}$ & $\begin{array}{l}\begin{array}{l}\text { Scheduling board \& activity } \\
\text { sequence chart; transition }\end{array} \\
\text { warning; } \\
\text { preferred \& nonterspersing } \\
\text { tasks; change in seating; } \\
\text { teaching to ask for help }\end{array}$ \\
\hline $\begin{array}{l}6 . \\
\text { Christensen } \\
\text { et al. (2012) }\end{array}$ & 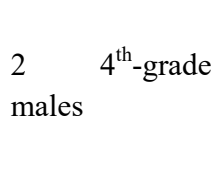 & At-risk & $\begin{array}{l}\text { Yelling; talking; out of } \\
\text { seat; playing with } \\
\text { items; off-task }\end{array}$ & $\begin{array}{l}\text { Peer \& teacher } \\
\text { attention }\end{array}$ & \begin{tabular}{l}
\multicolumn{3}{l}{ Contingency adjustment; social } \\
skills training; positive \\
reinforcement; \\
reinforcement
\end{tabular} \\
\hline $\begin{array}{l}\text { 7. Clarke et } \\
\text { al. (1995) }\end{array}$ & $\begin{array}{ll}3 \quad \text { boys }^{c} ; & 1 \\
5 \text {-yrs. old, } & 2 \\
\text { 11-yrs old. } & \end{array}$ & $\begin{array}{l}\text { emotionally } \\
\text { disturbed; } 1 \\
\text { ADHD }\end{array}$ & $\begin{array}{l}\text { Aggression; talking } \\
\text { without permission, } \\
\text { noise-making, leaving } \\
\text { seat; property } \\
\text { destruction, } \\
\text { noncompliance }\end{array}$ & Escape & $\begin{array}{l}\text { Modifying interest level of } \\
\text { tasks/assignments }\end{array}$ \\
\hline $\begin{array}{l}\text { 8. Dejager \& } \\
\text { Filter }(2015)\end{array}$ & $\begin{array}{l}3 \quad \text { boys; } \\
\text { kindergarten, } \\
4^{\text {th }} \text { grade, } 5^{\text {th }} \\
\text { grade }\end{array}$ & At-risk & $\begin{array}{l}\text { Off-task; arguing; } \\
\text { blurting out; disruptive } \\
\text { verbalizations }\end{array}$ & $\begin{array}{l}\text { Escape; } \\
\text { attention }\end{array}$ & $\begin{array}{lrr}\text { Physical } & \text { boundary } \\
\text { identification } & \& & \text { visual } \\
\text { cues/prompts; } & \text { replacement } \\
\text { behavior } & \text { training; } \\
\text { noncontingent } & \text { reinforcement; } \\
\text { breaks from tasks; }\end{array}$ \\
\hline
\end{tabular}




\begin{tabular}{|c|c|c|c|c|c|}
\hline $\begin{array}{l}\text { 9. Dunlap, } \\
\text { White, Vera, } \\
\text { Wilson, \& } \\
\text { Panacek } \\
\text { (1996) }\end{array}$ & $\begin{array}{l}1 \quad \text { boy } \quad(7-y r . \\
\text { old, } 2^{\text {nd }} \text { grade); } \\
2 \text { girls }(1 \quad 7-y r . \\
\text { old } \quad \text { second } \\
\text { grade; } 19-\text { yr. } \\
\left.\text { old } 4^{\text {th }} \text { grade }\right)\end{array}$ & $\begin{array}{l}\text { Emotionally } \\
\text { disturbed }\end{array}$ & $\begin{array}{l}\text { Property destruction; } \\
\text { negative } \\
\text { verbalizations; leaving } \\
\text { seat without } \\
\text { permission; } \\
\text { aggression; not follow } \\
\text { directions }\end{array}$ & Escape & $\begin{array}{l}\text { Modified assignments: shorter } \\
\text { worksheets, enlarged print on } \\
\text { worksheets, highlighting } \\
\text { words; student choice of } \\
\text { worksheets }\end{array}$ \\
\hline $\begin{array}{l}\text { 10. Dwyer et } \\
\text { al. }(2012)\end{array}$ & $\begin{array}{l}3 \text { boys ( } 2 \text { 7-yrs. } \\
\text { old, } 18 \text {-yrs } \\
\text { old.) }\end{array}$ & $\begin{array}{l}\text { Emotionally } \\
\text { disturbed }\end{array}$ & $\begin{array}{l}\text { Talking out; out of } \\
\text { seat; moving paper on } \\
\text { desk; staring; not } \\
\text { following directions }\end{array}$ & Escape & $\begin{array}{l}\text { Replacement behavior training: } \\
\text { ask for help, ask for a break, } \\
\text { ask for either help or a break }\end{array}$ \\
\hline $\begin{array}{l}\text { 11. Edwards } \\
\text { et al. (2002) }\end{array}$ & 10-yr. old boy & ADHD & $\begin{array}{lr}\text { Off-task, } & \text { physical } \\
\text { aggression, } & \text { throwing } \\
\text { objects, not } & \text { following } \\
\text { instructions } & \end{array}$ & $\begin{array}{l}\text { Attention } \\
\text { (functional analysis) }\end{array}$ & $\begin{array}{l}\text { Positive } \\
\text { attention-extinction/planned } \\
\text { ignoring }\end{array}$ \\
\hline
\end{tabular}

\begin{tabular}{|c|c|c|c|c|c|}
\hline $\begin{array}{l}\text { 12. Ellis \& } \\
\text { Magee } \\
(1999)\end{array}$ & $\begin{array}{l}3 \quad \text { boys: } 2 \\
10 \text {-yrs. old } 5^{\text {th }} \\
\text { graders; } 16-\text { yr. } \\
\text { old } \\
\text { kindergarten }\end{array}$ & At-risk & $\begin{array}{lr}\text { Physical } & \text { aggression; } \\
\text { yelling; } & \text { tantrums; } \\
\text { property } & \text { destruction; } \\
\text { not } & \text { following } \\
\text { instructions } & \end{array}$ & $\begin{array}{l}\text { Escape; attention } \\
\text { (functional analysis) }\end{array}$ & $\begin{array}{l}\text { Task alteration (e.g., shortening } \\
\text { work assignments); novel } \\
\text { materials; extinction, academic } \\
\text { tutoring, token reinforcement }\end{array}$ \\
\hline $\begin{array}{l}\text { 13. Filter \& } \\
\text { Horner } \\
(2009)\end{array}$ & $\begin{array}{l}24^{\text {th }} \text { grade } \\
\text { males }\end{array}$ & $\begin{array}{l}\text { Learning } \\
\text { disability; } \\
\text { at-risk }\end{array}$ & $\begin{array}{l}\text { Out of seat; talking } \\
\text { out; poking peers; }\end{array}$ & $\begin{array}{l}\text { Escape } \\
\text { (functional analysis) }\end{array}$ & $\begin{array}{l}\text { Presenting material on } \\
\text { audiotape; multiple choice for } \\
\text { comprehension questions; } \\
\text { functional communication } \\
\text { training; contingent access to } \\
\text { tasks }\end{array}$ \\
\hline $\begin{array}{l}\text { 14. Grady \& } \\
\text { Peck (1997) }\end{array}$ & $\begin{array}{l}6 \text {-yr. old male, } \\
1 \text { st grade }\end{array}$ & At-risk & $\begin{array}{l}\text { Poking peers; kicking } \\
\text { feet; out of seat; } \\
\text { talking }\end{array}$ & $\begin{array}{l}\text { Adult attention } \\
\text { (functional analysis) }\end{array}$ & $\begin{array}{l}\text { Self-monitoring \& adult } \\
\text { contingent attention }\end{array}$ \\
\hline $\begin{array}{l}\text { 15. Hansen } \\
\text { et al. (2014) }\end{array}$ & male $4^{\text {th }}$ grader & At-risk & $\begin{array}{l}\text { Talking to others; } \\
\text { making } \\
\text { inappropriate } \\
\begin{array}{l}\text { moises; } \\
\text { movements }\end{array}\end{array}$ & $\begin{array}{l}\text { Attention } \\
\text { (functional analysis) }\end{array}$ & $\begin{array}{l}\text { Self-monitoring; } \\
\text { teacher attention }\end{array}$ \\
\hline $\begin{array}{l}\text { 16. Haydon } \\
(2012)\end{array}$ & $\begin{array}{l}\text { male } 11-y r s . \\
\text { old; } 5^{\text {th }} \text { grader }\end{array}$ & $\begin{array}{l}\text { Learning } \\
\text { disability }\end{array}$ & $\begin{array}{l}\text { Touching or talking to } \\
\text { peers; singing; taking } \\
\text { objects from peers; } \\
\text { making noises }\end{array}$ & Escape & $\begin{array}{l}\text { Reduction in difficulty level of } \\
\text { work }\end{array}$ \\
\hline $\begin{array}{l}\text { 17. Hoff et } \\
\text { al. }(2005)\end{array}$ & $\begin{array}{l}\text { male } 12-y r s . \\
\text { old; } 6^{\text {th }} \text { grade }\end{array}$ & At-risk & $\begin{array}{l}\text { Talking to peers; } \\
\text { making animal noises; } \\
\text { making faces; touching } \\
\text { peers; throwing } \\
\text { materials; out of seat }\end{array}$ & $\begin{array}{l}\text { Peer attention \& } \\
\text { escape (multiply } \\
\text { controlled) }\end{array}$ & $\begin{array}{l}\text { Preferred materials and } \\
\text { contingent peer attention (i.e., } \\
\text { sitting clos to preferred peers) }\end{array}$ \\
\hline $\begin{array}{l}\text { 18. Ingram } \\
\text { et al. (2005) }\end{array}$ & $26^{\text {th }}$ grade boys & $\begin{array}{l}1 \text { at-risk. } 1 \\
\text { ADHD }\end{array}$ & $\begin{array}{l}\text { Not following } \\
\text { directions; difficulty } \\
\text { beginning work }\end{array}$ & Escape & $\begin{array}{l}\text { Ask for help by raising hand; } \\
\text { given breaks; differential } \\
\text { negative reinforcement of } \\
\text { alternative behavior (DNRA) }\end{array}$ \\
\hline
\end{tabular}


Out of seat; arguing;

taunting, name calling; Teacher \& peer

19. Kamps 7-yr. old girl \& et al. (2006) 7-yr. old boy

At-risk

noncompliance; attention \& escape

aggression (e.g., (multiply

hitting, kicking, controlled)

pushing, throwing (functional analysis)

objects)
1 boy 8 -yrs. old

in $4^{\text {th }}$ grade 1 et al. (2001) in $1^{\text {st }}$ grade $\&$ 1girl 6-yrs. old in $1^{\text {st }}$ grade emotional disorder

21. Kern et 2 boys 11-yrs. \&

al. (2001) old in $5^{\text {th }}$ grade behavioral disorder
20. Kennedy boy 6-yrs. old

ADHD \&

\section{Hitting/kicking peers;}

swe

objects; Adult \& peer Person-centered planning; swearing; attention; escape increased contingent teacher noncompliance; (multiply) attention; specifying rules; obscene gestures; controlled) self-monitoring yelling

\section{Making noises; talking} out; profanity; leaving Escape work area; not (functional analysis) selection and completion; following directions

Hitting or kicking

peers; noncompliance;

22. Lane, 6 yr. old

Smither et kindergarten At-risk al. (2007) boy

Preferred medium for task interesting activity
Self-monitoring; differential reinforcement; positive teacher note to student out of seat; talking to Attention

peers; making faces;

touching objects praise; ignoring inappropriate behaviors; self-monitoring;
23. Lane et 1 boy \& 1 girl $\begin{array}{ll}\text { 23. Lane et } & \text { both in } 2^{\text {nd }} \text { At-risk } \\ \text { al. (2006) } & \begin{array}{l}\text { grade } \\ \end{array}\end{array}$
Talking to peers; out of seat; blurting out; Teacher \& peer compartment to place playing with materials; attention materials; checklist addressing behavioral goals; positive

Teach \& prompt replacement behaviors; providing reinforcement

\begin{tabular}{|c|c|c|c|c|c|}
\hline $\begin{array}{l}24 . \quad \text { Lane, } \\
\text { Weisenbach } \\
\text { et al. (2007) }\end{array}$ & $\begin{array}{l}1 \text { boy } \& 1 \text { girl } \\
\text { both } 7 \text {-yrs. old } \\
\text { in } 2^{\text {nd }} \text { grade }\end{array}$ & At-risk & $\begin{array}{l}\text { Talking to peers; } \\
\text { making negative } \\
\text { statements; rolling } \\
\text { eyes; out of assigned } \\
\text { area; blurting out }\end{array}$ & $\begin{array}{l}\begin{array}{l}\text { Teacher \& peer } \\
\text { attention } \\
\text { escape (boy) }\end{array} \\
\text { (girl); }\end{array}$ & $\begin{array}{l}\text { Replacement behavior training; } \\
\text { changing seating arrangement; } \\
\text { prompt cards; contingent } \\
\text { reinforcement; } \\
\text { contingencies; DNRA. }\end{array}$ \\
\hline $\begin{array}{l}\text { 25. LeGray } \\
\text { et al. (2010) }\end{array}$ & $\begin{array}{l}\text { 6-yr. old boy in } \\
\text { kindergarten }^{\mathrm{d}}\end{array}$ & At-risk & $\begin{array}{l}\text { Inappropriate } \\
\text { vocalizations, }\end{array}$ & $\begin{array}{l}\text { Access to tangible } \\
\text { objects } \\
\text { (functional analysis) }\end{array}$ & $\begin{array}{l}\text { Differential reinforcement of } \\
\text { other behavior (DRO); } \\
\text { differential reinforcement of a } \\
\text { functional alternative (DRA) }\end{array}$ \\
\hline $\begin{array}{l}\text { 26. Lo \& } \\
\text { Cartledge } \\
(2006)\end{array}$ & $\begin{array}{l}4 \text { boys }(7 \quad \& \\
8 \text {-yr. olds in } 2^{\text {nd }} \\
\text { grade; } 2 \text {-yr. } \\
\text { olds, } 1 \text { in } 3^{\text {rd }} \\
\text { grade, } 1 \text { in } 4^{\text {th }} \\
\text { grade }\end{array}$ & $\begin{array}{l}2 \text { at-risk; } 2 \\
\text { ADHD }\end{array}$ & $\begin{array}{lr}\text { Not following } \\
\text { directions; } \\
\text { away from assing } \\
\text { task/materials; playing } \\
\text { with objects, talking to } \\
\text { peers }\end{array}$ & Adult attention & $\begin{array}{l}\text { Differential reinforcement of } \\
\text { incompatible behavior (DRI); } \\
\text { DRA; } \\
\text { contingent positive teacher } \\
\text { attention }\end{array}$ \\
\hline $\begin{array}{l}\text { 27. Luiselli } \\
\& \quad \text { Pine } \\
(1999)\end{array}$ & $\begin{array}{l}\text { 10-yr. old } 3^{\text {rd }} \\
\text { grade girl }\end{array}$ & ADHD & $\begin{array}{l}\text { Taking objects without } \\
\text { permission; finding } \\
\text { others' possessions at } \\
\text { her desk }\end{array}$ & Adult attention & $\begin{array}{l}\text { Token economy; returning } \\
\text { stolen object; notes home }\end{array}$ \\
\hline $\begin{array}{l}\text { 28. Maag \& } \\
\text { Larson } \\
(2004)\end{array}$ & $\begin{array}{l}2 \text { boys in } 5^{\text {th }} \\
\text { grade }\end{array}$ & $\begin{array}{l}1 \text { boy with } \\
\text { EBD, } 1 \\
\text { with } \\
\text { learning } \\
\text { disability }\end{array}$ & $\begin{array}{l}\text { Verbal comments \& } \\
\text { noises }\end{array}$ & $\begin{array}{l}\text { Peer \& } \\
\text { attention }\end{array}$ & $\begin{array}{l}\text { Preferred seating; contingent } \\
\text { reinforcement }\end{array}$ \\
\hline
\end{tabular}




\begin{tabular}{|c|c|c|c|c|c|}
\hline $\begin{array}{l}\text { 29. Moore et } \\
\text { al. }(2005)\end{array}$ & $\begin{array}{l}6 \text {-yr. old } 1^{\text {st }} \\
\text { grade boy }\end{array}$ & At-risk & $\begin{array}{l}\text { Looking around the } \\
\text { room; not following } \\
\text { teacher directions; } \\
\text { giggling; talking; out } \\
\text { of seat; touching others }\end{array}$ & Esc & $\begin{array}{l}\text { Reducing task duration; } \\
\text { modifying assignments (e.g., } 3 \\
\text { worksheets of } 5 \text { problems } \\
\text { instead of } 1 \text { worksheet of } 15 \\
\text { problems) }\end{array}$ \\
\hline $\begin{array}{l}30 . \\
\text { Newcomer } \\
\& \quad \text { Lewis } \\
(2004)\end{array}$ & $\begin{array}{l}2 \text { boys }(9-\mathrm{yr} . \\
\text { old } 3^{\text {rd }} \text { grade; } \\
11-y r . \text { old } 5^{\text {th }} \\
\text { grade }) 1 \text { girl } \\
11-y r s . \text { old in } \\
5^{\text {th }} \text { grade }\end{array}$ & At-risk & $\begin{array}{lr}\text { Aggression; off-task; } \\
\text { not following } \\
\text { directions; challenging } \\
\text { teachers }\end{array}$ & $\begin{array}{l}\text { Escape; attention } \\
\text { (functional analysis) }\end{array}$ & $\begin{array}{l}\text { Contingent reinforcement; } \\
\text { extinction; change task } \\
\text { difficulty; altering antecedents }\end{array}$ \\
\hline $\begin{array}{l}31 . \\
\text { Packenham } \\
\text { et al. (2004) }\end{array}$ & 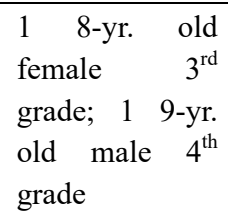 & At & $\begin{array}{l}\text { Looking at other } \\
\text { students; fidgeting } \\
\text { with objects; talking to } \\
\text { peers, out of seat; } \\
\text { laughing }\end{array}$ & $\begin{array}{l}\text { Teacher attention; } \\
\text { escape }\end{array}$ & $\begin{array}{l}\text { Contingent teacher attention for } \\
\text { appropriate behavior; provide } \\
\text { explicit instructions; shorten } \\
\text { lessons; positive reinforcement }\end{array}$ \\
\hline $\begin{array}{l}\text { 32. Patterson } \\
(2009)\end{array}$ & $\begin{array}{l}15-\text { yr. old male } \\
9^{\text {th }} \text { grade }\end{array}$ & At-risk & $\begin{array}{l}\text { Walking around the } \\
\text { room }\end{array}$ & Teacher attention & $\begin{array}{l}\text { Teacher spending } 2 \text { min. } \\
\text { talking with student prior to } \\
\text { lesson }\end{array}$ \\
\hline $\begin{array}{l}\text { 33. Payne, } \\
\text { Scott, \& } \\
\text { Conroy } \\
(2007)\end{array}$ & $\begin{array}{l}2 \text { females }(10 \\
\& \text { 11-yr. old } 3^{\text {rd }} \\
\text { grade }) \\
\text { males }(9 \text { yr. old } \\
4^{\text {th }} \text { grade, } 11-y r . \\
\left.\text { old } 5^{\text {th }} \text { grade }\right)\end{array}$ & At-risk & $\begin{array}{l}\text { Talking or gesturing to } \\
\text { peer; looking away } \\
\text { from teacher or } \\
\text { academic materials; } \\
\text { not following } \\
\text { directions; } \\
\text { manipulating objects }\end{array}$ & $\begin{array}{l}\text { Teacher \& peer } \\
\text { attention; escape }\end{array}$ & $\begin{array}{l}\text { Negative reinforcement (e.g., } \\
\text { earn a break for task } \\
\text { completion); spend time with a } \\
\text { peer contingent on task } \\
\text { completion; } \\
\text { reinforcement; }\end{array}$ \\
\hline $\begin{array}{l}\text { 34. Preciado, } \\
\text { Horner, \& } \\
\text { Baker } \\
(2009)\end{array}$ & $\begin{array}{l}3 \text { males }\left(22^{\text {nd }}\right. \\
\text { grade, } 14^{\text {th }} \\
\text { grade }) 1 \text { female } \\
3^{\text {rd }} \text { grade }\end{array}$ & $\begin{array}{l}\text { Learning } \\
\text { disability; } \\
\text { at-risk }\end{array}$ & $\begin{array}{l}\text { Staring out window; } \\
\text { playing with objects; } \\
\text { talking with peers; out } \\
\text { of seat }\end{array}$ & $\begin{array}{l}\text { Escape } \\
\text { (functional analysis) }\end{array}$ & $\begin{array}{l}\text { Teaching decoding } \text { skills; } \\
\text { reviewing/previewing } \\
\text { content } \\
\text { instructions; teaching social } \\
\text { skills }\end{array}$ \\
\hline $\begin{array}{lr}\text { 35. } & \text { Radford } \\
\& & \text { Ervin } \\
(2002) & \end{array}$ & A male & $\mathrm{AD}$ & $\begin{array}{l}\text { Punching/kicking } \\
\text { peers; talking back; } \\
\text { throwing objects }\end{array}$ & & $\begin{array}{l}\text { Adult proximity to student; } \\
\text { teach social skills as } \\
\text { replacement behavior }\end{array}$ \\
\hline $\begin{array}{l}\text { 36. } \\
\text { Sanford } \\
\& \quad \text { Horner } \\
(2012)\end{array}$ & $\begin{array}{l}2 \text { males }(7-y r . \\
\text { old } 2^{\text {nd }} \text { grade, } \\
8 \text {-yr. old } 3^{\text {rd }} \\
\text { grade) } \& \quad 2 \\
\text { females } \quad(9-y r . \\
2^{\text {nd }} \text { grade, } 9-y r . \\
\left.3^{\text {rd }} \text { grade }\right)\end{array}$ & At-risk & $\begin{array}{l}\text { Talking out; out of } \\
\text { seat; playing with } \\
\text { objects; not following } \\
\text { directions; hair pulling }\end{array}$ & $\begin{array}{l}\text { Escape } \\
\text { (functional analysis) }\end{array}$ & $\begin{array}{l}\text { Instruction-level } \\
\text { placement }\end{array}$ \\
\hline $\begin{array}{l}\text { 37. Shumate } \\
\& \quad \text { Wills } \\
(2010)\end{array}$ & $\begin{array}{l}2 \text { boys }(7 \quad \& \\
8 \text {-yr. old }) 1 \text { girl } \\
7 \text {-yr. old }) \text { all } 2^{\text {nd }} \\
\text { grade }\end{array}$ & At-risk & $\begin{array}{l}\text { Arguing, taunting, } \\
\text { name calling, \& } \\
\text { singing; } \quad \text { pencil } \\
\text { tapping; talking to } \\
\text { peers; out of seat }\end{array}$ & $\begin{array}{l}\text { Teacher attention } \\
\text { (functional analysis) }\end{array}$ & DRO \& DRA \\
\hline $\begin{array}{l}\text { 38. Skinner } \\
\text { et al. }(2009)\end{array}$ & $1^{\text {st }}$ grade male & ADHD & $\begin{array}{l}\text { Making noises; } \\
\text { whistling; talking out; } \\
\text { crawling; tipping chair; } \\
\text { banging foot on floor; } \\
\text { hitting, pushing, } \\
\text { throwing objects }\end{array}$ & $\begin{array}{l}\text { Escape } \\
\text { (functional analysis) }\end{array}$ & $\begin{array}{l}\text { DRA; DNRA; contingent } \\
\text { reinforcement; token economy }\end{array}$ \\
\hline
\end{tabular}




\begin{tabular}{|c|c|c|c|c|c|}
\hline $\begin{array}{l}\text { 39. Smith \& } \\
\text { Sugai (2000) }\end{array}$ & $\begin{array}{l}\text { 13-yr. old } 7^{\text {th }} \\
\text { grade male }\end{array}$ & EBD & $\begin{array}{l}\text { Talking out; out of } \\
\text { seat; hitting others; Peer \& } \\
\text { looking away from attention } \\
\text { activity }\end{array}$ & teacher & $\begin{array}{l}\text { Self-monitoring work, } \\
\text { comments to peers, \& raising } \\
\text { hand and waiting to be called } \\
\text { on }\end{array}$ \\
\hline
\end{tabular}

\begin{tabular}{|c|c|c|c|c|c|}
\hline $\begin{array}{l}\text { 40. Stichter, } \\
\text { Lewis, } \\
\text { Johnson, \& } \\
\text { Trussell } \\
\text { (2004) }\end{array}$ & $\begin{array}{l}7 \text {-yr. old } 2^{\text {nd }} \\
\text { grade male }\end{array}$ & $\begin{array}{l}\text { EBD } \\
\text { ADHD }\end{array}$ & $\begin{array}{l}\text { Talking without } \\
\text { permission; no } \\
\text { physical orientation or } \\
\text { involvement in activity }\end{array}$ & $\begin{array}{l}\text { Teacher attention } \\
\text { (functional analysis) }\end{array}$ & 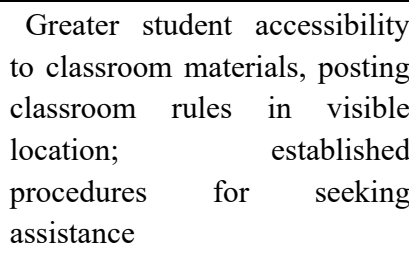 \\
\hline $\begin{array}{l}\text { 41. Storey et } \\
\text { al. (1994) }\end{array}$ & $\begin{array}{l}\text { 6-yr. old } \\
\text { kindergarten } \\
\text { boy }\end{array}$ & At-risk & $\begin{array}{l}\text { Talking out; touching } \\
\text { others; } \quad \text { throwing } \\
\text { objects; taunting peers }\end{array}$ & $\begin{array}{l}\text { Peer \& teacher } \\
\text { attention }\end{array}$ & $\begin{array}{l}\text { Contingent teacher attention for } \\
\text { appropriate behavior; } \\
\text { self-monitoring }\end{array}$ \\
\hline $\begin{array}{l}\text { 42. Trussell } \\
\text { et al. (2016) }\end{array}$ & $\begin{array}{l}3 \text { males }(17-y r . \\
\text { old } 2^{\text {nd }} \text { grade; } 1 \\
8 \text {-yr. old } 3^{\text {rd }} \\
\text { grade; } 19-y r . \\
\left.\text { old } 4^{\text {th }} \text { grade }\right)\end{array}$ & At-risk & $\begin{array}{ll}\text { Talking \& blurting out; } \\
\text { throwing } & \text { objects; } \\
\text { hitting peers; } & \text { playing } \\
\text { with objects; } & \text { crying; } \\
\text { sexually } & \text { explicit } \\
\text { language } & \end{array}$ & Attention; escape & $\begin{array}{l}\text { Teaching replacement } \\
\text { behaviors; social skills training; } \\
\text { contingent attention; ignore } \\
\text { student for inappropriate } \\
\text { behavior; repositioning desks; } \\
\text { providing breaks; }\end{array}$ \\
\hline $\begin{array}{l}\text { 43. Trussell, } \\
\text { Lewis, } \\
\text { Stichter. } \\
(2008)\end{array}$ & $\begin{array}{l}3 \text { males }(17-y r . \\
\text { old } 1^{\text {st }} \text { grade; } 1 \\
11-y r . \text { old } 5^{\text {th }} \\
\text { grade; } 1 \quad 8-y r . \\
\left.\text { old } 3^{\text {rd }} \text { grade }\right)\end{array}$ & $\begin{array}{l}\text { Emotional } \\
\text { disturbance; } \\
\text { learning } \\
\text { disability }\end{array}$ & $\begin{array}{l}\text { Cry; argue; yell; verbal } \\
\text { threats; not following } \\
\text { directions; throwing } \\
\text { chairs; knocking over } \\
\text { tables; hitting; kicking } \\
\text { peers }\end{array}$ & $\begin{array}{l}\text { Attention; escape } \\
\text { (functional analysis) }\end{array}$ & $\begin{array}{l}\text { Put exemplar student work on } \\
\text { display; teach hand raising; } \\
\text { create schedule; increase } \\
\text { information covered; teacher } \\
\text { feedback; contingent positive } \\
\text { reinforcement }\end{array}$ \\
\hline $\begin{array}{l}\text { 44. Umbreit } \\
\text { (1995) }\end{array}$ & $\begin{array}{l}8 \text {-yr. old } 3^{\text {rd }} \\
\text { grade boy }\end{array}$ & ADHD & $\begin{array}{l}\text { Talking to peers; facial } \\
\text { or hand gestures; } \\
\text { walking around room; } \\
\text { tapping pencil }\end{array}$ & $\begin{array}{l}\text { Escape \& peer } \\
\text { attention (multiply } \\
\text { controlled) } \\
\text { (functional analysis) }\end{array}$ & $\begin{array}{l}\text { Change seating arrangement } \\
\text { away from friends; request a } \\
\text { break; ignore inappropriate } \\
\text { behavior }\end{array}$ \\
\hline
\end{tabular}

\footnotetext{
a many studies indicated using a "multi-element" design. These designs were used during functional analysis (i.e., testing hypothesis), whereas the present review was only interested in the subsequent design used to analyze the efficacy of the intervention developed from the FBA.

${ }^{b}$ there was a second participant but diagnosed with a pervasive developmental disorder.

${ }^{c}$ there were four boys but only three were include in the analysis because the fourth had autism.

${ }^{d}$ there were two additional boys but both were 4 years old and, consequently, excluded for not meeting inclusion criteria.
}

\subsection{Design Features}

The majority of SCRDs were reversal $(n=16)$ and multiple baseline $(n=14)$. Other designs used were a simple $\mathrm{AB}(n=7)$, alternating treatments $(n=3)$, changing conditions $(n=3)$, and multi-element $(n=1)$. Only designs that evaluated the efficacy of an intervention developed from the FBA were used. Many studies initially indicated using a multi-element design but those were for determining and testing the hypothesized functions and not the designs they used to assess the effectiveness of the interventions developed from them.

Dependent variables and identified function(s). The majority of studies targeted between three to five dependent variables. The three most commonly targeted behaviors were talking to others $(n=28)$, being out of seat/walking around $(n=27)$, and not following directions/noncompliance $(n=26)$. Some studies used fairly subjective terms such as aggression $(n=9)$ while others were more specific such as hitting $(n=5)$ and kicking $(n=$ 6). Two studies targeted sexually explicit comments (Trussell, Lewis, \& Raynor, 2016; Turton, Umbreit, \& Mathur, 2011).

There were two main obtained functions: attention $(n=25)$ and escape $(n=21)$. Six studies found attention and escape to be multiply controlled (Bessett \& Wills, 2007; Cho \& Blair, 2017; Hoff, Ervin, \& Friman, 2005; Kamps, Wendland, \& Culpepper, 2006; Kennedy et al., 2001; Umbreit, 1995). The only other function besides escape and 
attention was access to tangible objects (LeGray et al., 2010). There were 19 out of the 44 studies that conducted a functional analysis to verify hypothesized functions.

FBA developed interventions. Most studies $(n=38)$ developed multi-component interventions with only six using a one element intervention: differential reinforcement of other behavior (DRO; Broussard \& Northrup, 1997), modifying interest level of tasks/assignments (Clarke et al., 1995), reducing task difficulty (Haydon, 2012), teacher spending two minutes talking to student before a lesson (Patterson, 2009), and assigning more challenging tasks (Umbreit, 1995). The most common intervention components were teaching replacement behaviors, contingent attention, extinction, differential reinforcement of alternative behavior (DRA), differential negative reinforcement of alternative behavior (DNRA), self-monitoring, and rearranging antecedent cues for the occurrence of appropriate behavior. From the descriptions of the interventions, quite elaborate and complicated techniques were used to determine whether problems behaviors displayed by participants during academic-related activities were maintained by either attention or escape.

\subsection{Statistical Analysis}

Effects of studies. Effect sizes were calculated for $145 \mathrm{AB}$ contrasts and were then averaged for each study that appear in Table 2. Overall omnibus effect sizes for each type were as follows: SMD (mean $=2.26, \mathrm{SD}=1.266$, range $0.12-3.40) ; \operatorname{IRD}($ mean $=.7754, \mathrm{SD}=0.267$, range $=0-1)$; and Tau (mean $=.7712, \mathrm{SD}=0.272$, range $=0$ - 1). Results of independent samples t-tests were insignificant on all three effect size types for differential effectiveness of interventions based on whether a functional analysis was conducted to conform hypothesized functions versus those using only indirect measures: $\operatorname{IRD}(t=1.301, p=.09)$, Tau- $U(t=1.038, p=.15)$, and SMD $(t=0.983, p=.16)$. There also were no significant differences in the effectiveness of interventions based on either the function of attention versus escape for IRD $(t=-0.856, p=.19)$, Tau- $U(t=-0.750, p=.22)$, and SMD $(t=$ $-0.605, p=.27)$.

Conservative dual criterion. There were a total of $112 \mathrm{AB}$ contrasts that met evaluation criteria (i.e., too few intervention data points, alternating treatments design with no baseline). Based on the criteria developed by Fisher et al. (2003), $80(71 \%)$ of $\mathrm{AB}$ contrasts demonstrated systematic change while 32 (29\%) represented nonsystematic change.

Publication bias. To address the "file drawer effect," the number of studies with results of zero required to reduce the overall effect to insignificant or suspect levels was determined for SMD, IRD and Tau effect sizes. It would take an average of 219 cases each with an effect size of 0 to bring the overall SMD, IRD, and Tau, into small to ineffective ranges. There are typically between one and six participants in SCRD studies. Using an average of three participants, 73 "filed" studies (almost half as many as met inclusion criteria) would be needed to bring obtained effect sizes into the ineffective range.

Table 2. Mean Study Effect Sizes

\begin{tabular}{lllllll}
\hline \multicolumn{1}{l}{ Study } & \multicolumn{2}{l}{ Effect Sizes } & & & & \\
& IRD & & Tau & \multicolumn{2}{l}{ SMD } & \\
& Mean & SD & Mean & SD & Mean & SD \\
\hline 1. Bessett \& Wills (2007) & .76 & 0.127 & .77 & 0.218 & 1.74 & 0.784 \\
2. Broussard \& Northrup (1997) & .82 & 0.137 & .86 & 0.100 & 1.47 & 0.520 \\
3. Campbell \& Anderson (2008) & 0 & 0 & .22 & 0.097 & .08 & 0.015 \\
4. Carter \& Horner (2009) & .60 & 0.165 & .56 & 0.166 & 1.11 & 0.366 \\
5. Cho \& Blair (2017) & .98 & 0.036 & .99 & 0.010 & 3.40 & 1.216 \\
6. Christensen et al. (2012) & .55 & 0.050 & .37 & 0.365 & -.10 & 1.163 \\
7. Clark et al. (1995) & .71 & 0.234 & .73 & 0.236 & 2.1 & 0.962 \\
8. Dejager \& Filter (2015) & .44 & 0.415 & .51 & 0.350 & .60 & 4.63 \\
9. Dunlap et al. (1996) & .91 & 0.117 & .97 & 0.047 & 3.40 & 2.60 \\
10. Dwyer et al. (2012) & .61 & 0.204 & .82 & 0.105 & 2.03 & 0.429 \\
11. Edwards et al. (2002) & .84 & ----- & .95 & ----- & 3.06 & ---- \\
\hline
\end{tabular}




\begin{tabular}{|c|c|c|c|c|c|c|}
\hline 12. Ellis \& Magee (1999) & .85 & 0.070 & .91 & 0.026 & 2.69 & 0.607 \\
\hline 13. Filter \& Horner (2009) & .56 & 0.437 & .61 & 0.383 & .913 & 1.290 \\
\hline 14. Grady \& Peck (1997) & .76 & 0.329 & 73 & 0.377 & 1.99 & 1.596 \\
\hline 15. Hansen et al. $(2014)^{\mathrm{a}}$ & 1 & ----- & 1 & ---- & 3.40 & ----- \\
\hline 16. Haydon $(2012)^{\mathrm{a}}$ & 1 & ----- & 1 & ----- & 3.40 & ----- \\
\hline 17. Hoff et al. $(2005)^{\mathrm{a}}$ & 1 & ----- & 1 & ----- & 3.40 & ----- \\
\hline 18. Ingram et al. $(2005)^{\mathrm{a}}$ & 1 & ---- & 1 & ----- & 3.28 & ----- \\
\hline 19. Kamps et al. (2006) & .60 & 0.149 & .66 & 0.199 & 1.93 & 0.580 \\
\hline 20. Kennedy et al. (2001) & .75 & 0.248 & .67 & 0.364 & 1.61 & 1.482 \\
\hline 21. Kern et al. (2001) & .71 & 0.034 & .75 & 0.117 & 1.35 & 0.130 \\
\hline 22. Lane, Smither et al. (2007) & .58 & 0.083 & .74 & 0.120 & 1.33 & 0.001 \\
\hline 23. Lane et al. $(2006)^{\mathrm{a}}$ & 1 & ----- & 1 & ----- & 3.40 & ---- \\
\hline 24. Lane, Weisenbach et al. (2007) & 1 & 0 & 1 & 0 & 3.65 & 6.504 \\
\hline 25. LeGray et al. $(2010)^{b}$ & $\mathrm{n} / \mathrm{a}$ & $\mathrm{n} / \mathrm{a}$ & $\mathrm{n} / \mathrm{a}$ & $\mathrm{n} / \mathrm{a}$ & $\mathrm{n} / \mathrm{a}$ & $\mathrm{n} / \mathrm{a}$ \\
\hline 26. Lo \& Cartledge (2006) & .57 & 0.214 & .62 & 0.261 & 1.47 & 1.060 \\
\hline 27. Luiselli \& Pine (1999) ${ }^{\mathrm{a}}$ & .42 & ---- & .48 & ---- & 1.48 & ----- \\
\hline 28. Maag \& Larson (2004) & 1 & 0 & 1 & 0 & 3.88 & 0.006 \\
\hline 29. Moore et al. (2005) & .83 & 1.110 & .78 & 0.128 & 2.85 & 0.502 \\
\hline 30. Newcomer \& Lewis (2004) & .64 & 0.135 & .59 & 0.230 & 1.52 & 0.778 \\
\hline 31. Packenham et al. (2004) & 1 & ----- & 1 & ----- & 3.40 & ----- \\
\hline 32. Patterson (2009) ${ }^{\mathrm{a}}$ & 1 & ---- & 1 & ---- & 3.40 & ----- \\
\hline 33. Payne et al. (2007) & 1 & 0 & 1 & 0 & 3.40 & 4.068 \\
\hline 34. Preciado et al. (2009) & .76 & 0.223 & .77 & 0.159 & 2.46 & 1.271 \\
\hline 35. Radford \& Ervin (2002) & .80 & 0.200 & .73 & 0.267 & 1.56 & 1.028 \\
\hline 36. Sanford \& Horner (2012) & .82 & 0.164 & .71 & 0.203 & 1.79 & 0.863 \\
\hline 37. Shumate \& Wills (2010) & .90 & 0.119 & .84 & 0.132 & 3.07 & 1.135 \\
\hline 38. Skinner et al. (2009) & 1 & ----- & 1 & ----- & 3.40 & ----- \\
\hline 39. Smith \& Sugai $(2000)^{\mathrm{a}}$ & .75 & ----- & .87 & ----- & 1.85 & ----- \\
\hline 40. Stichter et al. (2004) $)^{\mathrm{a}}$ & 0 & ---- & 0 & ---- & .304 & ----- \\
\hline 41. Storey et al. (1994) & 1 & ----- & 1 & ----- & 3.40 & ----- \\
\hline 42. Trussell et al. (2016) & .72 & 2.90 & .73 & 0.217 & 1.65 & 0.660 \\
\hline 43. Trussell et al. (2008) & .52 & 0.113 & .64 & 0.214 & 1.27 & 0.637 \\
\hline 44. Umbreit (1995) & 1 & 0 & 1 & 0 & 3.40 & 1.66 \\
\hline
\end{tabular}

${ }^{\mathrm{a}}$ only one participant.

${ }^{\mathrm{b}}$ alternating treatment design without baseline.

\subsection{Characteristics of the Data}

It was not possible to calculate $\mathrm{CDC}$ lines for 44 of the $\mathrm{AB}$ contrasts due to fewer than five data points in intervention phases. Specifically, there were 20 intervention phases with four data points, 18 with three data points, five with two data points, and one with 1 intervention data point. Many of the baselines had very unstable trends. (e.g., Besset \& Wills, 2007; Broussard \& Northrup, 1997; Cho \& Blair, 2017; Edwards, Magee, \& Ellis, 2002; Kern, Ringdahl, Hilt, \& Sterling-Turner, 2001).

There were other peculiarities with the data, especially related to measurement and quantity of dependent variables. 
There were studies that had very low baseline levels. These low levels were sometimes related to the dependent variable, such as aggression (e.g., Bessett \& Wills, 2007) which tends to be a low frequency low duration but high intensity behavior. Some studies had five or fewer episodes of the target behavior during baseline (e.g., Cho \& Blair, 2017) or low percentages of intervals, with one average baseline level being $6 \%$ (Dejager \& Filter, 2015). Three studies had $\mathrm{Y}$ axis numbers of $0,0.5,1,1.5,2,2.5$, and 3 for episodes of the dependent variable during one minute observations (e.g., Christensen et al., 2012; Haydon, 2012; Luiselli \& Pine, 1999). It is difficult to interpret a 0.5 disruptive behavior. Another curiosity was the dependent variable of "out of seat" being recorded with frequency instead of duration or interval recording (e.g., Patterson, 2009).

\subsection{Social Validity}

A little less than half the studies $(n=20$ [44\%]) addressed social validity in terms of having teachers rate their satisfaction with interventions developed from the FBAs. The most common way to assess social validity was through surveys and questionnaires that typically had Likert-scale ratings. Two studies interviewed teachers to determine social validity (Ingram et al., 2005; Moore et al., 2005). Eight (18\%) studies included an explicitly stated social validity goal (Broussard \& Northrup, 1997; Dejager \& Filter, 2015; Kamps et al., 2006; Lane et al., 2006; Lane, Weisenbach, Phillips, \& Wehby, 2007; Packenham et al., 2004; Shumate \& Wills, 2010; Skinner et al., 2009). All but one study (Dejager \& Filter, 2015) stated the impact of the intervention related to the stated goal. Only two studies employed social comparison peers (Hansen et al., 2014; Lo \& Cartledge, 2006).

In terms of interventionists, teachers were the sole agent in nine studies, 13 were researchers, and 16 were both teacher and researcher. In the latter, the researcher(s) typically conducted the FBA and/or functional analysis and then teachers were trained to implement the FBA-based intervention. For studies that identified just teachers as the agent, they were trained to conduct both the FBA and intervention. There were six studies in which the intervention agent was a paraeducator, school staff, graduate students, or therapist (Bessett \& Wills, 2007; Campbell \& Anderson, 2008; Edwards et al., 2002; Ellis \& Magee, 1999; Hansen et al., 2014; Haydon, 2012).

\section{Discussion}

The present meta-analysis reviewed the literature on the use of FBAs with youth in kindergarten through $12^{\text {th }}$ grade who either had high incidence disabilities or were at risk for and displayed challenging behaviors in classroom settings during academic related tasks/activities. Calculated effect sizes were commensurate to those obtained by other reviewers (e.g., Bruni et al., 2017; Losinski et al., 2014; Miller \& Lee, 2013). No previous review calculated $\mathrm{CDC}$ lines in order to determine the percentage of systematic change ( $71 \%$ of AB contrasts), and only $40 \%$ of studies reported social validity. There were no significant differences in effectiveness of interventions based on whether or not a functional analysis was conducted nor whether the controlling function was escape or attention.

\subsection{Descriptive Analysis}

Several conclusions can be reached from the descriptive analysis. First, most participants did not have any disabilities nor psychiatric disorders but rather displayed challenging behaviors in the classrooms of general education teachers. These participants were typically males with a mean age of approximately nine years. Second, most of the studies were conducted in general education classrooms during academic lessons, activities, or tasks. Third, the most typical types of behaviors targeted were talking to others, being out of seat/walking around, and those under the category of defiance and refusal to follow directions. Fourth, the identified functions, except in one case, were either attention or escape, and quite elaborate and complicated techniques were used to make these determinations, sometimes for only one participant. Fifth, most interventions based on the FBAs were teaching replacement behaviors, positive reinforcement for appropriate behaviors and extinction for inappropriate behaviors, rearranging antecedents, DNRA (e.g., giving students whose behaviors were maintained by escape breaks for task completion), and self-monitoring.

The use of FBAs has been considered best practice in schools generally-especially those using multi-tiered systems of support (MTSS) because they follow a universal supports paradigm that addresses struggling students regardless of the presence or absence of a disability. However, certain instances - particularly in the United States for students with disabilities served under the individuals with disabilities education act (IDEA) - mandate its use (Individuals with Disabilities Education Act, 2004). Specifically, FBAs must be conducted for behaviors that interfere with the learning environment, for students who are suspended for more than ten schools days, when misconduct results in a manifestation determination, or when weapons, drugs, or serious bodily injury occurs (Katsiyannis \& Maag, 2001). Nevertheless, as school continue to adopt MTSS frameworks, they will be required to build capacity among staff in function-based thinking and assessment for students across tiers of support within both general and special education settings. 


\subsection{FBA Effectiveness}

All previous meta-analyses obtained effect sizes in acceptable or effective ranges using a variety of calculations, although some with large variability and heterogeneity (e.g., Common et al., 2017). It is difficult to draw conclusions regarding intervention effectiveness from SCRD effect sizes due to presence of autocorrelations, lack of independence between observations, and the tendency of some calculations (i.e., SMD) to overestimate results (Campbell, 2004; Hershberger, Wallace, Green, \& Marquis, 1999; Olive \& Smith, 2005).

In the present review both baseline and intervention data could be characterized as moderately to highly unstable which does not help interpret effect sizes. Specifically, baselines were characterized by few data points, low numbers/percentages, and highly unstable trends. Collectively, these are serious methodological flaws in interpreting SCRD results (Kazdin, 2010).

Perhaps these data problems were reflective of studies conducted in natural environments, such as the general education classroom, and that more stable data and observations may be obtained for studies conducted in more controlled settings such as clinics, residential facilities, or psychiatric hospitals. Lang, Sigafoos, Lancioni, Didden and Rispoli (2010) reviewed a small set of studies conducted in different settings and concluded that in some instances FBA procedures were similar between settings and others they were different.

\subsection{Social Validity}

It is somewhat surprising, given the acceptance of using FBAs and interventions generated from them, that less than half the studies (44\%) addressed social validity. However, $32 \%$ of studies had researchers or graduate students conducting all aspects of the FBA process which would make it moot to assess social validity, and also reflect the complexity of the process and levels of expertise required for implementation. The researcher and teacher collaborated in 13 of the studies but only six collected measures of social validity. In these instances the teacher implemented the function-based intervention that the researchers developed.

The issue is not that general education teachers are incapable of learning and implementing FBAs (e.g., Maag \& Larson, 2004; Moore et al., 2002; Packenham et al., 2004), but whether they will use this methodology, consistently, with fidelity, and independently when not being observed - especially if the procedures are perceived to be time-consuming and require high levels of expertise. For example, Lane, Smither, et al. (2007) engaged in six activities to determine that for one participant the function of his behavior was peer and adult attention: (a) Preliminary Functional Assessment Survey, (b) functional assessment interview with the student, (c) 10 hours of direct observation using an A-B-C approach, (d) teacher completed Motivation Assessment Scale, (e) teacher version of the Social Skills Rating System, and (f) the School Archival Record Search. These measures would be a daunting undertaking for any teacher, let alone considering many studies conducted actual experimental manipulations to confirm hypothesized function using multi-component designs (e.g., Broussard \& Northup, 1997; Clarke et al., 1995; Dwyer, Rozewiski, \& Simonsen, 2012; Edwards et al., 2002). It is difficult to imagine school personnel—even school psychologists — would have the expertise and time to engage in these activities for one student.

One positive result related to social validity was that interventions based on FBAs were no more effective when functional analyses were conducted than those using only indirect measures. Both direct and indirect measures have advantages and disadvantages, but indirect measures in which conducting functional analyses are unnecessary may be more social acceptable if teachers were aware of their existence and use. For example, Dufrene, Kazmerski and Labrot (2017) reviewed the social validity of indirect FBA instruments and concluded that teachers and other school personal have very limited knowledge of their existence and relevance. This result corroborates the need for more extensive and varied social validity measures to be included in FBA intervention studies for youth with high incidence disabilities or those simply displaying challenging behaviors.

Another mixed conclusion involves the socially validity of interventions developed from FBAs. In the present review, except for replacement behavior training and perhaps self-monitoring, the remaining interventions were simple procedures such as rearranging antecedents, providing positive reinforcement, changing assignment difficulty, or giving students who misbehave breaks contingent on completing certain portions of their work. These simple techniques are based on elementary principles of applied behavior analysis included in special education teacher preparation programs but rarely part of general education curriculum. For example, Merrett and Wheldall (1993) interviewed 176 secondary school teachers regarding their professional training and behavior management. Nearly three-quarters of them were dissatisfied with the preparation in this area while a majority indicated interest in attending training courses in behavior management. Gebbie, Ceglowski, Taylor, and Miels, (2012) found that when classroom teachers of preschool children with disabilities were surveyed, their most frequent request was 
how to address students' challenging behaviors. Ironically, it is not a stretch to imagine that if more general education teachers in the studies reviewed possessed these rudimentary behavior management skills that some of the participants identified as displaying challenging behaviors would not even be included.

A final issue related to social validity was that only surveys and questionnaires typically involving Likert-type scales were used and, consequently, may limit the amount and type of information they offer (Leko, 2014). Several researchers have expanded on the social validity construct by using qualitative approaches in intervention research such as interviews (e.g., Broer, Doyle, \& Giangreco, 2005; Copeland et al., 2004; Gerber \& Popp, 1999; Leko, 2014; Lyst, Gabriel, O'Shaughnessy, Meyers, \& Meyers, 2005). In the present meta-analysis 16 studies had both researchers and teachers jointly developed and conducted the FBA and subsequent interventions. These studies would have been an excellent format in which to conduct interviews between researchers and teachers.

\section{Conclusion}

There is no question that FBAs and interventions created from them are an essential component in the education and treatment of individuals with moderate to severe disabilities. Further, the results of the present review corroborate those obtained by Common et al. (2017) but extent them to the corpus of literature extending back to the 1980s. It is not surprising that the two main functions obtained from the reviewed studies were escape and attention. The studies were conducted in mostly general education classrooms during academic lessons and tasks - typically during independent paper-and-pencil seatwork. Some students with high-incidence disabilities or those at risk display academic deficits and, therefore, may find instructional lessons and the task demands that accompany them aversive and act out to escape or gain attention from peers. The question remains whether intensive multi-component FBAs such as those conducted in the reviewed studies are necessary and even possible for educators who may lack the expertise for doing so. However, when the interventionist was a teacher or other educational personnel, they tended to find the interventions developed from the FBA acceptable in terms of their social validity.

\section{References}

Baer, D. M., Wolf, M. M., \& Risley, T. (1968). Some current dimensions of applied behavior analysis. Journal of Applied Behavior Analysis, 1, 91-97. https://doi.org/10.1901/jaba.1968.1-91

Bessett, K. K., \& Wills, H. P. (2007). An example of an elementary school paraprofessional-implemented functional analysis and intervention. Behavioral Disorders, 32, 192-210.

Broer, S. M., Doyle, M. B., \& Giangreco, R. F. (2005). Perspectives of students with intellectual disabilities about their experience with paraprofessional support. Exceptional Children, 71, 415-430.

Broussard, C., \& Northrup, J. (1997). The use of functional analysis to develop peer interventions for disruptive classroom behavior. School Psychology Quarterly, 12, 65-76. https://doi.org/10.1037/h0088948

Bruni, T. P., Drevon, D., Hixson, M., Wyse, R., Corcoran, S., \& Fursa, S. (2017). The effect of functional behavior assessment on school-based interventions: A meta-analysis of single-case research. Psychology in the Schools, 54, 351-369. https://doi.org/10.1002/pits.22007

Campbell, A., \& Anderson, C. M. (2008). Enhancing effects of check-in/check-out with function-based support. Behavioral Disorders, 33, 233-245. https://doi.org/10.1177/019874290803300404

Campbell, J. M. (2004). Statistical comparison of four effect sizes for single-subject designs. Behavior Modification, 28, 234-246. https://doi.org/10.1177/0145445503259264

Carr, E. G. (1977). The motivation of self-injurious behavior: A review of some hypotheses. Psychological Bulletin, 84, 800-816. https://doi.org/10.1037//0033-2909.84.4.800

Carter, D., \& Horner, R. H. (2009). Adding function-based behavioral support to First Step to Success: Integrating individualized and manualized practices. Journal of Positive Behavior Interventions, 11, 22-34. https://doi.org/10.1177/1098300708319125

Cho, S.-J., \& Blair, K.-S. C. (2017). Using a multicomponent function-based intervention to support students with attention deficit hyperactivity disorder. The Journal of Special Education, 50, 227-238. https://doi.org/10.1177/0022466916655186

Christensen, L., Renshaw, T. L., Caldarella, P., \& Young, J. R. (2012). Training a general educator to use function-based support for students at risk for behavior disorders. Education, 133, 313-335.

Clarke, S., Dunlap, G., Foster-Johnson, L., Childs, K. E., Wilson, D., White, R., \& Vera, A. (1995). Improving the conduct of students with behavioral disorders by incorporating student interests into curricular activities. 
Behavioral Disorders, 20, 221-237. https://doi.org/10.1177/019874299502000402

Cohen, J. (1988). Statistical power for the behavioral sciences (2nd ed.). Hillsdale, NJ: Erlbaum.

Common, E. A., Lane, K. L., Pustejovsky, J. E., Johnson, A. H., \& Johl, L. E. (2017). Functional assessment-based interventions for students with or at-risk for high-incidence disabilities: Field testing single-case synthesis methods. Remedial and Special Education, 38, 331-352. https://doi.org/10.1177/0741932517693320

Copeland, S. R, Hughes, C., Carter, E. W., Guth, C., Presley, J. A., Williams, C. R., \& Fowler, S. E. (2004). Increasing access to general education: Perspectives of participants in a high school peer support program. Remedial and Special Education, 25, 343-352. https://doi.org/10.1177/074193325040250060201

Dejager, B. W., \& Filter, K. J. (2015). Effects of prevent-teach-reinforce on academic engagement and disruptive behavior. Journal Applied School Psychology, 31, 369-391. https://doi.org/10.1080/15377903.2015.1084966

Delfs, C., \& Campbell, J. (2010). A quantitative synthesis of developmental disability research: the impact of functional assessment methodology on treatment effectiveness. The Behavior Analyst Today, 11, 4-19. https://doi.org/10.1037/h0100685

Dufrene, B. A., Kazmerski, J. S., \& Labrot, A. (2017). The current status of indirect functional assessment instruments. Psychology in the Schools, 54, 331-350. https://doi.org/10.1002/pits.22006

Dunlap, G., White, R., Vera, A., Wilson, D., \& Panacek, L. (1996). The effects of multi-component, assessment based curricular modifications on the classroom behavior of children with emotional and behavioral disorders. Journal of Behavioral Education, 6, 481-500. https://doi.org/10.1007/BF02110518

Dwyer, K., Rozewiski, D., \& Simonsen, B. (2012). A comparison of function-based replacement behaviors for escape-motivated students. Journal of Emotional and Behavioral Disorders, 20, 115-125. https://doi.org/10.1177/1063426610387432

Edwards, W. H., Magee, S. K., \& Ellis, J. (2002). Identifying the effects of idiosyncratic variables on functional analysis outcomes: A case study. Education and Treatment of Children, 25, 317-330.

Ellis, J., \& Magee, S. K. (1999). Determination of environmental correlates of disruptive classroom behaviour: Integration of functional analysis into public school assessment process. Educational \& Treatment of Children, 22, 291-316.

Ervin, R. A., Radford, P. M., Bertsch, K., Piper, A. L., Ehrhardt, K. E., \& Poling, A. (2001). A descriptive analysis and critique of the empirical literature on school-based functional assessment. School Psychology Review, 30, 193-210.

Filter, K. J., \& Horner, R. H. (2009). Function-based academic interventions for problem behavior. Education and Treatment of Children, 32, 1-19. https://doi.org/10.1353/etc.0.0043

Fisher, W. W., Kelley, M. E., \& Lomas, J. E. (2003). Visual aids and structured criteria for improving visual inspection and interpretation of single-case designs. Journal of Applied Behavior Analysis, 36, 387-406. https://doi.org/10.1901/jaba.2003.36-387

Fuchs, D., Fuchs, L., \& Bahr, M. (1990). Mainstream assistance teams: A scientific basis for the art of consultation. Exceptional Children, 57, 128-139.

Gage, N. A., Lewis, T. J., \& Stichter, J. P. (2012). Functional behavioral assessment-based interventions for students with or at risk for emotional and/or behavioral disorders in school: A hierarchical linear modeling meta-analysis. Behavioral Disorders, 37, 55-77. https://doi.org/10.32469/10355/10347

Gebbie, D. H., Ceglowski, D., Taylor, L. K., \& Miels, J. (2012). The role of teacher efficacy in strengthening classroom support for preschool children with disabilities who exhibit challenging behaviors. Early Childhood Education Journal, 40, 35-46. https://doi.org/10.1007/s10643-011-0486-5

Gerber, P. A., \& Popp, P. A. (1999). Consumer perspectives on the collaborative teaching model: Views of students with and without LD and their parents. Remedial and Special Education, 20, 288-296. https://doi.org/10.1177/074193259902000505

Goh, A. E., \& Bambara, L. M. (2012). Individualized positive support in school settings: A meta-analysis. Remedial and Special Education, 33, 271-286. https://doi.org/10.1177/0741932510383990

Grady, S. E., \& Peck, S. M. (1997). The use of functional assessment and self-management with a first grader. Child \& Family Behavior Therapy, 19, 29-43. https://doi.org/10.1300/J019v19n02 03 
Gresham, F. M. (2003). Establishing the technical adequacy of functional behavioral assessment: Conceptual and measurement challenges. Behavioral Disorders, 28, 282-298. https://doi.org/10.1177/019874290302800305

Hansen, B. D., Wills, H. P., Kamps, D. M., \& Greenwood, C. R. (2014). Effects of function-based self-management interventions. Journal of Emotional and Behavioral Disorders, 22, 149-159. https://doi.org/10.1177/1063426613476345

Haydon, T. (2012). Using functional behavior assessment to match task difficulty for a 5 th grade student: A case study. Education and Treatment of Children, 35, 459-476. https://doi.org/10.1353/etc.2012.0019

Hershberger, S. L., Wallace, D. D., Green, S. B., \& Marquis, J. G. (1999). Meta-analysis of single-case designs. In R. H. Hoyle (Ed.), Statistical strategies for small sample research (pp. 109-132). Newbury Park, CA: Sage.

Hoff, K. E., Ervin, R. A., \& Friman, P. C. (2005). Refining functional behavioral assessment: Analyzing the separate and combined effects of hypothesized controlling variables during ongoing classroom routines. School Psychology Review, 34, 45-57.

Horner, R. H., Swaminathan, H., Sugai, G., \& Smolkowski, K. (2012). Considerations for the systematic analysis of single-case research. Education and Treatment of Children, 35, 269-290. https://doi.org/10.1353/etc.2012.0011

Individuals with Disabilities Education Act. (2004). H. R. 1350, 108th Cong.

Ingram, K., Lewis-Palmer, T., \& Sugai, G. (2005). Function-based intervention planning: Comparing the effectiveness of FBA function-based and non-function-based intervention plans. Journal of Positive Behavior Interventions, 7, 224-236. https://doi.org/10.1177/10983007050070040401

Iwata, B. A., Dorsey, M. F., Slifer, K. J., Bauman K. E., \& Richman, G. S. (1982). Toward a functional analysis of self-injury. Analysis and Intervention in Developmental Disabilities, 2, 3-20. https://doi.org/10.1016/0270-4684(82)90003-9

Kamps, D., Wendland, M., \& Culpepper, M. (2006). Active teacher participation in functional behavior assessment for students with emotional and behavioral disorders risks in general education classrooms. Behavioral Disorders, 31, 128-146. https://doi.org/10.1177/019874290603100203

Katsiyannis, A., \& Maag, J. W. (2001). Manifestation determination as a golden fleece. Exceptional Children, 68, 85-96. https://doi.org/10.1177/0014402900106800105

Kazdin, A. E. (2010). Single-case research designs: Methods for clinical and applied settings (2nd ed.). New York, NY: Oxford University Press.

Kennedy, C. H., Long, T., Jolivette, K., Cox, J., Tang, J. C., \& Thompson, T. (2001). Facilitating general education participation for students with behavior problems by linking positive behavior supports and person-centered planning. Journal of Emotional and Behavioral Disorders, 9, 161-171. https://doi.org/10.1177/106342660100900302

Kern, L., Ringdahl, J. E., Hilt, A., \& Sterling-Turner, H. E. (2001). Linking self-management procedures to $\begin{array}{lllll}\text { functional analysis } & \text { results. Behavioral Disorders, 26, 226. }\end{array}$ https://doi.org/10.1177/019874290102600304

Lane, K. L., Smither, R., Huseman, R., Guffey, J., \& Fox, J. (2007). Function-based intervention to decrease disruptive behavior and increase academic engagement. Journal of Early and Intensive Behavior Interventions, 4, 348-364. https://doi.org/10.1037/h0100348

Lane, K. L., Weisenbach, J. L., Little, A., Phillips, A., \& Wehby, J. H. (2006). Illustrations of function-based interventions implemented by general education teachers: Building capacity at the school site. Education and Treatment of Children, 29, 549-571.

Lane, K. L., Weisenbach, J. L., Phillips, A., \& Wehby, J. H. (2007). Designing, implementing, and evaluating function-based interventions using a systematic, feasible approach. Behavioral Disorders, 32, 122-139. https://doi.org/10.1177/019874290703200205

Lang, R., Sigafoos, J, Lancioni, G., Didden, R., \& Rispoli, M. (2010). Influence of assessment setting on the results of functional analyses of problem behavior. Journal of Applied Behavior Analysis, 43, 565-567. https://doi.org/10.1901/jaba.2010.43-565

LeGray, M. W., Dufrene, B. A., Sterling-Turner, H., Olmi, D. J., \& Bellone, K. (2010). A comparison of function-based differential reinforcement interventions for children engaging in disruptive classroom 
behavior. Journal of Behavioral Education, 19, 185-204. https://doi.org/10.1007/s10864-010-9109-2

Leko, M. M. (2014). The value of qualitative methods in social validity research. Remedial and Special Education, 35, 275-286. https://doi.org/10.1177/0741932514524002

Lo, Y. Y., \& Cartledge, G. (2006). FBA and BIP: Increasing the behavior adjustment of African American boys in school. Behavioral Disorders, 31, 147-161. https://doi.org/10.1177/019874290603100204

Losinski, M., Maag, J. W., Katsiyannis, A., \& Ennis, R. P. (2014). Examining the effects and quality of interventions based on the assessment of contextual variables: A meta-analysis. Exceptional Children, 80, 407-422. https://doi.org/10.1177/0014402914527243

Luiselli, J. K., \& Pine, J. (1999). Social control of childhood stealing in a public school: A case study. Journal of Behavior Therapy and Experimental Psychiatry, 30, 231-239. https://doi.org/10.1016/S0005-7916(99)00023-3

Lyst, A. M., Gabriel, S., O'Shaughnessy, T. E., Meyers, J., \& Meyers, B. (2005). Social validity: Perceptions of check and connect with early literacy support. Journal of School Psychology, 43, 197-218. https://doi.org/10.1016/j.jsp.2005.04.004

Maag, J. W. (2014). Persistent issues in behavioral theory and practice. In L. Florian (Ed.), Handbook of special education (2nd ed., pp. 281-298). London, England: Sage.

Maag, J. W., \& Larson, P. J. (2004). Training a general education teacher to apply functional assessment. Education and Treatment of Children, 27, 26-36.

Maggin, D. M., Briesch, A. M., \& Chafouleas, S. M. (2013). An application of the What Works Clearinghouse standards for evaluating single-subject research: Synthesis of the self-management literature base. Remedial and Special Education, 34, 44-58. https://doi.org/10.1037/e304782003-001

McKenna, J. W., Flower, A., \& Adamson, R. (2016). A systematic review of function-based replacement behavior interventions for students with and at risk for emotional and behavioral disorders. Behavior Modification, 40, 678-712. https://doi.org/10.1177/014544551621489

McKenna, J. W., Flower, A., Kim, M. K., Ciullo, S., \& Haring, C. (2015). A systematic review of function-based interventions for students with learning disabilities. Learning Disabilities Research \& Practice, 30, 15-28. https://doi.org/10.1111/ldrp.12049

Merrett, F., \& Wheldall, K. (1993). How to teachers learn to manage classroom behaviour? A study of teachers' opinions about their initial training with special reference to classroom behavior management. Educational Studies, 19, 91-106. https://doi.org/10.1111/ldrp.12049

Miller, F. G., \& Lee, D. L. (2013). Do functional behavior assessments improve intervention effectiveness for students diagnosed with ADHD? A single-subject meta-analysis. Journal of Behavioral Education, 22, 253-282. https://doi.org/10.1007/s10864-013-9174-4

Mitchell, M. (2002). Engauge Digitizer (version 4.1) [computer software]. Retrieved from http://sourceforge.net/projects/digitizer/

Moore, D. W., Anderson, A., \& Kumar, K. (2005). Instructional adaptation in the management of escape-maintained behavior in a classroom. Journal of Positive Behavior Interventions, 7, 216-223. https://doi.org/10.1177/10983007050070040301

Moore, J. W., Edwards, R. P., Sterling-Turner, H. E., Riley, J., DuBard, M., \& McGeorge, A. (2002). Teacher acquisition of functional analysis methodology. Journal of Applied Behavior Analysis, 35, 73-77.

Nagler, E. M., Rindskopf, D. M., \& Shadish, W. R. (2008). Analyzing data from small $N$ designs using multilevel models: A procedural handbook. Washington, D C: U.S. Department of Education.

Nelson, J. R., Roberts, M. L., Mathur, S. R., \& Rutherford, R. B., Jr. (1999). Has public policy exceeded our knowledge base? A review of the functional behavioral assessment literature. Behavioral Disorders, 24, 169-179. https://doi.org/10.1177/019874299902400207

Newcomer, L. L., \& Lewis, T. J. (2004). Functional behavioral assessment: An investigation of assessment reliability and effectiveness of function-based interventions. Journal of Emotional and Behavioral Disorders, 12, 168-181. https://doi.org/10.1177/10634266040120030401

Olive, M. L., \& Smith, B. W. (2005). Effect size calculations and single subject designs. Educational Psychology, 25, 313-324. https://doi.org/10.1080/0144341042000301238 
Packenham, M., Shute, R., \& Reid, R. (2004). A truncated functional behavioral assessment procedure for children with disruptive classroom behaviors. Education and Treatment of Children, 27, 9-25.

Parker, R. I., Vannest, J., \& Brown, L. (2009). The improvement rate difference for single-case research. Exceptional Children, 75, 135-150. https://doi.org/10.1177/001440290907500201

Patterson, S. T. (2009). The effects of teacher-student small talk on out-of-seat behavior. Education and Treatment of Children, 32, 167-174. https://doi.org/10.1353/etcl0.0048

Payne, L. D., Scott, T. M., \& Conroy, M. (2007). A school-based examination of the efficacy of function-based intervention. Behavioral Disorders, 32, 158-173.

Preciado, J. A., Horner, R. H., \& Baker, S. K. (2009). Using a function-based approach to decrease problem behaviors and increase academic engagement for Latino English language learners. The Journal of Special Education, 42, 227-240. https://doi.org/10.1177/0022466907313350

Radford, P. M., \& Ervin, R. A. (2002). Employing descriptive functional assessment methods to assess low-rate, high-intensity behaviors: A case example. Journal of Positive Behavior Interventions, 4, 146-155. https://doi.org/10.1177/10983007020040030301

Rosenberg, M. S., Adams, D. C., \& Gurevitch, J. (2000). Metawin. Sunderland, MA: Sinauer.

Rosenthal, R. (1979). The "file drawer problem" and tolerance for null results. Psychological Bulletin, 86, 638-641. https://doi.org/10.1037/0033-2909.86.3.638

Sanford, A. K., \& Horner, R. H. (2012). Effects of matching instruction difficulty to reading level for students with escape-maintained problem behavior. Journal of Positive Behavior Interventions, 5, 79-89. https://doi.org/10.1177/1098300712449868

Sasso, G. M., Conroy, M. A., Stichter, J. P., \& Fox, J. J. (2001). Slowing down the bandwagon: The misapplication of functional assessment for students with emotional or behavioral disorders. Behavioral Disorders, 26, 282-296. https://doi.org/10.1177/019874290102600407

Scruggs, T. E., \& Mastropieri, M. A. (2012). PND at 25: Past, present, and future trends in summarizing single-subject research. Remedial and Special Education, 34, 9-19. https://doi.org/10.1177/0741932512440730

Shogren, K. A., Wehmeyer, M. L., \& Lane, K. L. (2016). Embedding interventions to promote self-determination

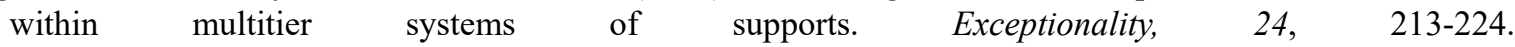
https://doi.org/10.1080/09362835.2015.1064421

Shumate, E., \& Wills, H. (2010). Classroom-based functional analysis and intervention for disruptive and off-task behaviors. Education and Treatment of Children, 33, 23-48. https://doi.org/10.1353/etc.0.0088

Skinner, J. N., Veerkamp, M. B., Kamps, D. M., \& Andra, P. R. (2009). Teacher and peer participation in functional analysis and intervention for a first grade student with attention deficit hyperactivity disorder. Education and Treatment of Children, 32, 243-266. https://doi.org/10.1353/etc.0.0059

Smith, B. W., \& Sugai, G. (2000). A self-management functional assessment-based behavior support plan for a middle school student with EBD. Journal of Positive Behavior Interventions, 2, 208-217. https://doi.org/10.1177/109830070000200405

Stage, S. A., \& Quiroz, D. R. (1997). A meta-analysis of interventions to decrease disruptive classroom behavior in public education settings. School Psychology Review, 26, 333-368.

Stichter, J. P., Lewis, T. J., Johnson, N., \& Trussell, R. (2004). Toward a structural assessment: Analyzing the merits of an assessment tool for a student with E/BD. Assessment for Effective Intervention, 30, 25-40. https://doi.org/10.1177/073724770403000103

Storey, K., Lawry, J. R., Ashworth, R., Danko, C. D., \& Strain, P. S. (1994). Functional analysis and intervention for disruptive behaviors of a kindergarten student. Journal of Educational Research, 87, 361-370. https://doi.org/10.1080/00220671.1994.9941267

Trussell, R. P., Lewis, T. J., \& Raynor, C. (2016). The impact of universal teacher practices and function-based behavior interventions on the rates of problem behaviors among at-risk students. Education and Treatment of Children, 39, 261-282. https://doi.org/10.1353/etc.2016.0012

Trussell, R. P., Lewis, T. J., \& Stichter, J. P (2008). The impact of targeted classroom interventions and function-based behavior interventions on problem behavior interventions on problem behaviors of students 
with emotional/behavioral disorders. Behavioral $\quad$ Disorders, $\quad 33$, 156. https://doi.org/10.1177/019874290803300303

Turton, A., Umbreit, J., \& Mathur, S. (2011). Systematic function-based interventions for adolescents with emotional and behavioral disorders in an alternative setting: Broadening the context. Behavioral Disorders, 36, 117-128.

Umbreit, J. (1995). Functional assessment and intervention in a regular classroom setting for the disruptive behavior of a student with attention deficit hyperactivity disorder. Behavioral Disorders, 20, $267-278$. https://doi.org/10.1177/019874299502000407

Umbreit, J, Ferro, J., Liaupsin, C., \& Lane, K. L. (2007). Functional behavioral assessment and function-based intervention: An effective, practical approach. Upper Saddle River, NJ: Prentice Hall.

Wolf, M. M. (1978). Social validity: The case for subjective measurements, or how behavior analysis is finding its heart. Journal of Applied Behavior Analysis, 11, 203-214. https://doi.org/10.1910/jaba.1978.11-203

\section{Copyrights}

Copyright for this article is retained by the author(s), with first publication rights granted to the journal.

This is an open-access article distributed under the terms and conditions of the Creative Commons Attribution license (http://creativecommons.org/licenses/by/4.0/). 